\title{
Long-term persistence of gastric dysbiosis after eradication of Helicobacter pylori in patients who underwent endoscopic submucosal dissection for early gastric cancer
}

\author{
Toshio Watanabe ${ }^{1}$ (1) - Yuji Nadatani ${ }^{1} \cdot$ Wataru Suda $^{2} \cdot$ Akira Higashimori $^{1} \cdot$ Koji Otani $^{1}$. Shusei Fukunaga ${ }^{1}$. \\ Shuhei Hosomi ${ }^{1}$. Fumio Tanaka ${ }^{1}$ - Yasuaki Nagami ${ }^{1} \cdot$ Koichi Taira $^{1} \cdot$ Tetsuya Tanigawa $^{1,3} \cdot$ Geicho Nakatsu $^{4}$. \\ Masahira Hattori ${ }^{2,5}$. Yasuhiro Fujiwara ${ }^{1}$
}

Received: 20 August 2020 / Accepted: 31 October 2020 / Published online: 17 November 2020

(c) The Author(s) 2020

\begin{abstract}
Background Gastric microbiome, other than Helicobacter pylori, plays a role in the tumorigenesis of gastric cancer (GC). Patients who undergo endoscopic submucosal dissection for early GC have a high risk of developing metachronous GC even after successful eradication of $H$. pylori. Thus, we investigated the microbial profiles and associated changes in such patients after the eradication of $H$. pylori.

Methods A total of $19 \mathrm{H}$. pylori-infected patients with early GC who were or to be treated by endoscopic resection, with paired biopsy samples at pre- and post-eradication therapy, were retrospectively enrolled. Ten $H$. pylori-negative patients were enrolled as controls. Biopsy samples were analyzed using 16S rRNA sequencing.

Results $H$. pylori-positive patients exhibited low richness and evenness of bacteria with the deletion of several genera, including Blautia, Ralstonia, Faecalibacterium, Methylobacterium, and Megamonas. H. pylori eradication partially restored microbial diversity, as assessed during a median follow-up at 13 months after eradication therapy. However, post-eradication patients had less diversity than that in the controls and possessed a lower abundance of the five genera mentioned above. The eradication of $\mathrm{H}$. pylori also altered the bacterial composition, but not to the same extent as that in controls. The microbial communities could be clustered into three separate groups: H. pylori-negative, pre-eradication, and post-eradication.

Conclusion Changes in dysbiosis may persist long after the eradication of $H$. pylori in patients with a history of GC. Dysbiosis may be involved in the development of both primary and metachronous GC after the eradication of $H$. pylori in such patients.
\end{abstract}

Keywords Dysbiosis · Gastric cancer · Helicobacter pylori $\cdot$ Eradication

Electronic supplementary material The online version of this article (https://doi.org/10.1007/s10120-020-01141-w) contains supplementary material, which is available to authorized users.

Toshio Watanabe

watanabet@med.osaka-cu.ac.jp

1 Department of Gastroenterology, Osaka City University Graduate School of Medicine, 1-4-3 Asahi-machi Abeno-ku, Osaka, Japan

2 RIKEN Center for Integrative Medical Sciences Laboratory for Microbiome Sciences, Yokohama, Kanagawa, Japan

\section{Introduction}

Helicobacter pylori was classified by the World Health Organization as a type I carcinogen in 1994 [1, 2]. Approximately, $89 \%$ of non-cardia gastric cancers (GCs), accounting for $78 \%$ of total GCs, are attributable to $H$. pylori infection [3]. Several randomized controlled trials have demonstrated a reduction

3 Department of Gastroenterology, Osaka City Juso Hospital, Osaka, Japan

4 Department of Immunology and Infectious Diseases/Genetics and Complex Diseases, Harvard T. H. Chan School of Public Health, Boston, MA, USA

5 Graduate School of Advanced Science and Engineering, Waseda University, Tokyo, Japan 
in the risk of developing primary and secondary GC after the removal of $H$. pylori [4-6]. A meta-analysis showed that the pooled ratio for the incidence of GC in individuals who underwent eradication therapy was 0.54 as compared to that in patients who were not treated for $H$. pylori [7]. These confirmed the preventive effect of $H$. pylori eradication on GC development. However, this effect is limited and some patients develop GC after the eradication of $H$. pylori. Several factors, such as endoscopic severe atrophy, histological intestinal metaplasia, and aberrant DNA methylation, in the gastric tissues, are associated with GC in $H$. pylori-eradicated patients [8-10]; however, their underlying mechanisms are unclear.

Animal studies using hypergastrinemic insulin-gastrin (INS-GAS) transgenic mice indicated that non- $H$. pylori microbiota play a crucial role in gastric carcinogenesis. INS-GAS-mice spontaneously developed atrophic gastritis, intestinal metaplasia, dysplasia, and GC [11], whereas germ-free INS-GAS mice had minimal to no gastric pathologies and did not develop GC. Monoassociation of H. pylori in germ-free INS-GAS mice accelerates gastritis and induces GC, but the severity of gastric lesions in these mice is less, and there is a delayed onset of GC compared to that in $H$. pylori-infected specific pathogen-free INS-GAS mice $[12,13]$. Recently, 16S rRNA-based metagenomic analysis revealed alterations in the composition and diversity of gastric microbiota in patients with $H$. pylori [14, 15]. Thus, dysbiosis may play an important role in gastric carcinogenesis.

Metachronous GC develops after the successful eradication of $H$. pylori with high incidence in patients after endoscopic submucosal dissection (ESD) for primary gastric neoplasms $[6,16]$. Thus, high-risk patients are suitable candidates for analysis of the pathogenesis of GC after the eradication of $H$. pylori. In this study, we investigated the alteration in microbiota upon the eradication of $H$. pylori in the gastric mucosa using (before and after eradication) paired samples from patients who underwent ESD for early GC.

\section{Methods}

\section{Patients}

The study cohort comprised $H$. pylori-infected patients diagnosed with early GC who planned to undergo ESD or were being followed up after ESD at the Osaka City University Hospital and underwent baseline endoscopy between June 2013 and December 2015. We retrospectively enrolled 21 patients with paired frozen biopsy samples during baseline and follow-up endoscopy (6 months or later) after the eradication of $H$. pylori. Patients who did not have gastric neoplasms or gastroduodenal ulcers (confirmed using endoscopy) were also included. H. pylori-naïve patients constituted the $H$. pylori-negative control group. The study had the following exclusion criteria during patient selection: prior documented treatment for $H$. pylori; use of antibiotics, proton pump inhibitors (PPIs), probiotics, or immunosuppressive drugs within 12 weeks of endoscopy; and coexistence of concomitant illnesses, including active malignant diseases except for GC, severe renal or hepatic dysfunction, and previous abdominal surgery. The study protocols were approved by the ethics committee of the Osaka City University Graduate School of Medicine (Approval No. 2413). Written informed consent was obtained from all participants.

\section{Determination of $\boldsymbol{H}$. pylori status}

H. pylori infection was determined using at least one of the three tests (serum antibody, histology, and ${ }^{13} \mathrm{C}$-urea breath test [UBT]). Successful eradication was concluded with negative readouts for histology and UBT $\geq 4$ weeks after the end of anti-H. pylori treatment. A subject was considered $H$. pylori-naïve if all of the following five criteria were met: (1) no history of $H$. pylori eradication; (2) seronegativity for $H$. pylori antibody; (3) negative UBT; (4) no pathological findings of gastric atrophy and intestinal metaplasia in biopsy specimens; and (5) none or mild gastric atrophy, as per the Kimura-Takemoto classification [17].

\section{H. pylori eradication therapies}

Post-ESD patients were administered $H$. pylori eradication therapies within 4 weeks of the baseline endoscopy, while patients with newly diagnosed gastric neoplasms underwent therapy within 12 weeks of ESD. H. pylori-positive individuals were administered first-line triple therapy consisting of a PPI (esomeprazole, lansoprazole, or rabeprazole) or vonoprazan in combination with amoxicillin plus clarithromycin (twice daily for 7 days). Patients who did not respond to the first-line therapy were administered second-line triple therapy where clarithromycin was replaced with metronidazole.

\section{Sample collection}

Biopsy specimens were obtained from all the patients from the greater curvature of the antrum and corpus of the stomach during baseline endoscopy. Gastric biopsy specimens were additionally obtained from $\mathrm{H}$. pylori-positive subjects who underwent ESD at least once after 6 months of successful eradication of $H$. pylori and were subjected to microbiome analysis. The latest specimens were analyzed for eradicated patients subjected to multiple biopsies. 


\section{DNA extraction and 16S rRNA gene amplicons sequencing}

DNA was enzymatically extracted from biopsies of gastric mucosa as previously described [18]. The V1-V2 region of the 16S rRNA was amplified using polymerase chain reaction (PCR) with the forward primer 27Fmod (5'- AAT GATACGGCGACCACCGAGATCTACACXxxxx xxxACACTCTTTCCCTACACGACGCTCTTCCGATCTagrgtttgatymtggctcag- $3^{\prime}$ ), containing the Illumina Nextera Adapters sequcence and a unique 8-bp barcode sequence for each sample (Barcode sequence by $\mathrm{x}$ ), and the reverse primer $338 \mathrm{R}$ (5'-CAAGCAGAAGACGGCATACGAGAT xxxxxxxxGTGACTGGAGTTCAGACGTGTGCTCTT CCGATCTtgctgcetccegtaggagt-3') containing the Illumina Nextera Adapters sequence using a 9700 PCR System (Life Technologies, Tokyo, Japan) as previously reported [19]. PCR amplicons were purified using AMPure XP magnetic purification beads (Beckman Coulter, Brea, CA, USA) and quantified using the Quant-iT PicoGreen dsDNA Assay Kit (Life Technologies Japan). An equal amount of each PCR amplicon was mixed and subjected to sequencing with MiSeq (Illumina) using MiSeq Reagent Kit v2 (500 cycles) according to the manufacturer's instructions [20].

\section{Processing of $16 \mathrm{~S}$ sequence data}

We analyzed the $16 \mathrm{~S}$ sequencing data using our previously reported method [20] with some modifications. Briefly, after demultiplexing the $16 \mathrm{~S}$ sequence reads, paired-end reads were joined using the fastq-join program. Reads with an average quality value of $<25$ and inexact matches to both universal primers were removed. Among the filter-passed reads, 3000 reads/sample were randomly chosen and analyzed. Subsequently, we sorted the selected reads using the average quality value and grouped them into operational taxonomic units (OTUs) using the UCLUST algorithm with a 97\% identity threshold. To reduce the effects of sequence error, a representative sequence of the OTUs was determined to be one having the highest redundancy in the OTU. Each OTU was aligned against the publicly available 16S (RDP and CORE) and NCBI genome databases using GLSEARCH. We used sequence similarity thresholds of $70 \%, 94 \%$, and $97 \%$ to assign the phylum, genus, and species, respectively.

\section{Statistical analysis}

Details of the statistical analyses are described in the Supplementary Methods (Supplemental Text).

\section{Results}

\section{Baseline patient characteristics}

Among the $21 \mathrm{H}$. pylori-positive patients with early GC, 15 were followed up after successful ESD. The remaining six patients were scheduled to undergo ESD at enrollment; subsequently, curative ESD was performed in all six patients. All of $21 \mathrm{GCs}$ were intestinal type. In 17 of the 21 patients, $H$. pylori was eradicated using first-line triple therapy, while four patients were subjected to second-line triple therapy to eradicate $H$. pylori. Gastric biopsy specimens after $H$. pylori eradication could not be obtained
Table 1 Baseline characteristics of patients

\begin{tabular}{llll}
\hline & $\begin{array}{l}\text { H. pylori-negative group } \\
(n=10)\end{array}$ & $\begin{array}{l}\text { Pre-eradication group } \\
(n=19)\end{array}$ & $p$ value \\
\hline $\begin{array}{l}\text { Age (years) } \\
\text { Median ( interquartile ranges) }\end{array}$ & $63.0(55.75-67.0)$ & $67.0(64.0-74.0)$ & 0.107 \\
Sex & 7 & 17 & 0.306 \\
Male & 3 & 2 & $<0.001$ \\
Female & & & \\
Severity of endoscopic atrophy & 10 & 0 & \\
None or mild (C-1 or C-2) & 0 & 11 & \\
Moderate (C-3 or O-1) & 0 & 8 & \\
Severe (O-2 or O-3) & & 0 & \\
Endoscopic diagnosis & 5 & 0 & \\
Normal & 1 & 0 & \\
Reflux esophagitis & 2 & $19(14 / 5)$ \\
Gastric ulcer scar & 2 & & \\
Fundic gland polyp & $0(0 / 0)$ & & \\
Early gastric cancer (past/present) & &
\end{tabular}


from one patient, and one patient was given a probiotic after the eradication of $H$. pylori. Therefore, these two patients were excluded from the study. We enrolled 13 $H$. pylori-negative (H. pylori-naïve) patients, but three were excluded from the study due to the use of a PPI. Table 1 shows the clinical characteristics of patients in the H. pylori-negative group and pre-eradication group (H. pylori-positive patients with early GC). There was no difference in the age and sex ratio of the patients between the two groups. The H. pylori-positive subjects had more severe endoscopic atrophy than that observed in the $H$. pylori-negative subjects.

\section{a}
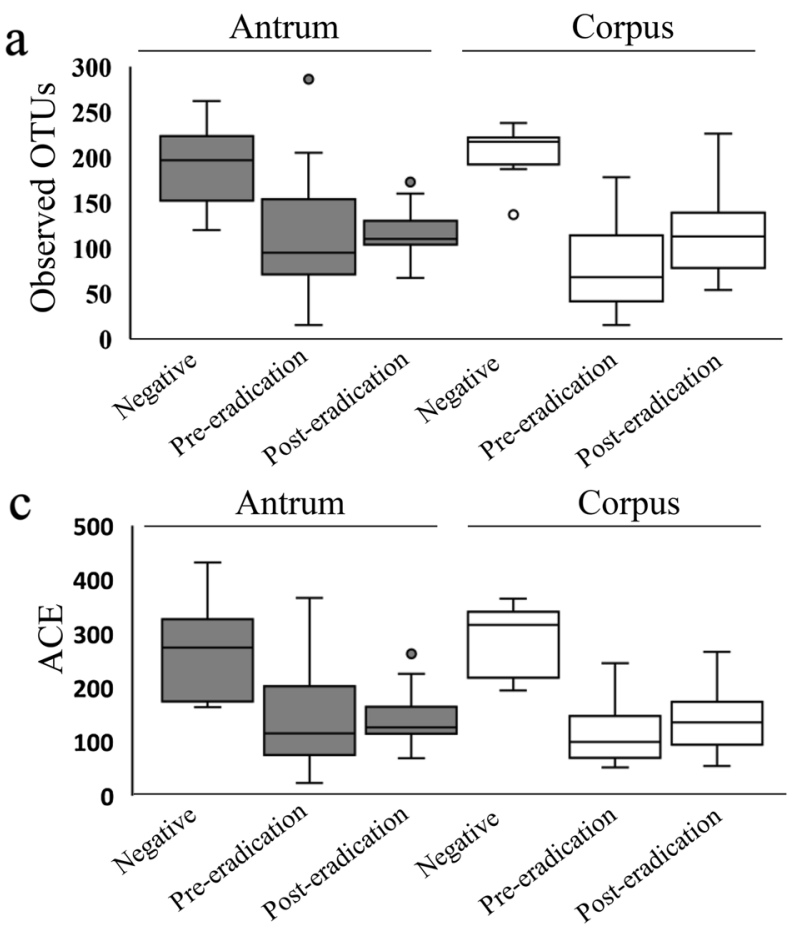

\section{Effect of $\boldsymbol{H}$. pylori infection on gastric bacterial diversity and richness}

We obtained paired (before and after $H$. pylori eradication) samples from the antrum and corpus from 18 out of $19 \mathrm{H}$. pylori-positive patients with early GC, while in one patient, paired samples were obtained from the antrum only. Samples were obtained from both sites for eight out of the $10 \mathrm{H}$. pylori-negative patients, while one sample from either antrum or corpus was obtained from two patients. We obtained a total of 2,261,850 highquality $16 \mathrm{~S}$ reads using the Illumina MiSeq platform
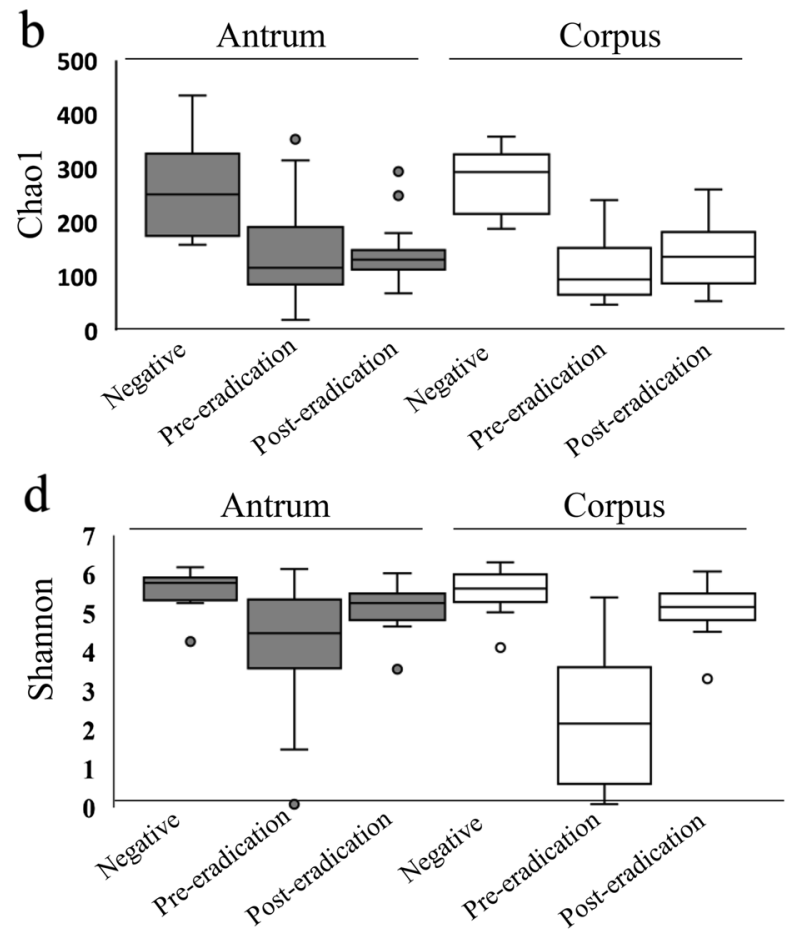

\begin{tabular}{lcccc}
\hline e & Observed OTUs & Chao1 & ACE & Shannon \\
\hline Antrum vs. Corpus & & & & \\
Negative & 0.945 & 0.945 & 0.945 & 0.641 \\
Pre-eradication & 0.024 & 0.130 & 0.142 & $<0.001$ \\
Post-eradication & 0.267 & 0.417 & 0.325 & 0.966 \\
\hline Negative vs. pre-eradication & & & & \\
Antrum & 0.002 & 0.006 & 0.004 & 0.002 \\
Corpus & $<0.001$ & $<0.001$ & $<0.001$ & $<0.001$ \\
\hline Negative vs. post-eradication & & & & \\
Antrum & $<0.001$ & $<0.001$ & $<0.001$ & 0.021 \\
Corpus & $<0.001$ & $<0.001$ & $<0.001$ & 0.041 \\
\hline Pre-eradication vs. post-eradication & & & & \\
Antrum & 0.983 & 0.891 & 0.891 & $<0.006$ \\
Corpus & 0.081 & 0.393 & 0.393 & $<0.001$ \\
\hline
\end{tabular}

Fig. 1 Comparison of $\alpha$-diversity in the gastric microbiome between different gastric regions or $H$. pylori status. The numbers of observed OTUs (a) and indices of Chaol (b), ACE (c), and Shannon (d) in each group have been expressed as medians and interquartile ranges. e The $P$ values between different groups are shown. Wilcoxon rank sum test was used to compare the values of $H$. pylori-negative patients with those of patients in the pre-eradication or post-eradication groups. The Wilcoxon signed-rank test was used to compare the difference in the paired samples from the pre- and post-H. pylori eradication groups 

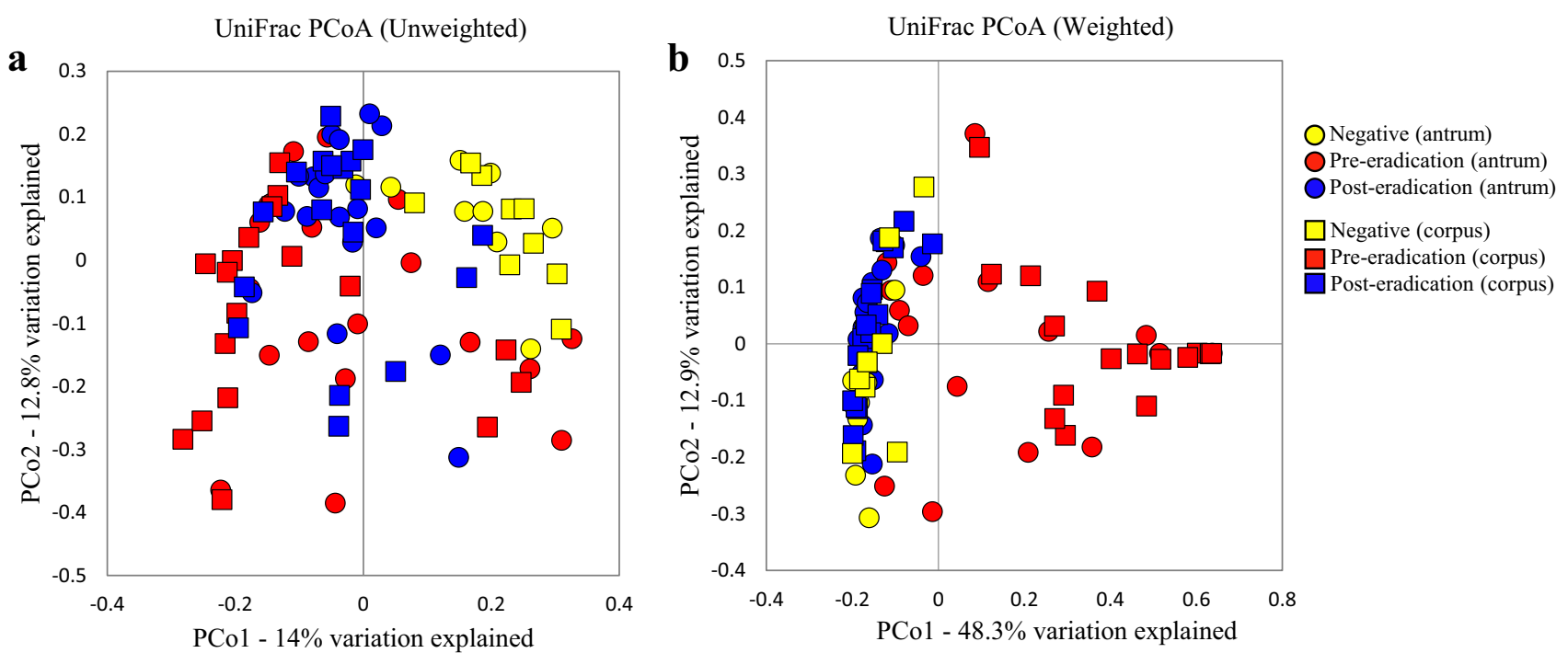

\begin{tabular}{lcccc}
\hline Group comparison & \multicolumn{2}{c}{ Unweighted UniFrac } & \multicolumn{2}{c}{ Weighted UniFrac } \\
& $\mathrm{R}^{2}$ & $P$ value & $\mathrm{R}^{2}$ & $P$ value \\
\hline Antrum vs. corpus & & & & \\
Negative & 0.053 & 0.698 & 0.093 & 0.115 \\
Pre-eradication & 0.039 & 0.108 & 0.167 & $<0.001$ \\
Post-eradication & 0.018 & 0.981 & 0.015 & 0.783 \\
\hline Negative vs. pre-eradication & & & & \\
Antrum & 0.088 & $<0.001$ & 0.155 & 0.005 \\
Corpus & 0.186 & $<0.001$ & 0.426 & $<0.001$ \\
\hline Negative vs. post-eradication & & & & \\
Antrum & 0.088 & $<0.001$ & 0.110 & 0.008 \\
Corpus & 0.471 & $<0.001$ & 0.132 & 0.307 \\
\hline Pre-eradication vs. Post-eradication & & & & \\
Antrum & 0.046 & 0.021 & 0.162 & $<0.001$ \\
Corpus & 0.064 & $<0.003$ & 0.390 & $<0.001$ \\
\hline
\end{tabular}

Fig. $2 \beta$-Diversity distances between different $H$. pylori groups using principal coordinate analysis (PCoA). a Unweighted UniFrac PCoA. b Weighted UniFrac PCoA. c PERMANOVA data compar-

(Supplemental Table 1). The Good's coverage indices of the 3000 reads per sample were $0.954( \pm 0.002)$ and $0.934( \pm 0.005)$ for total and $H$. pylori-removing analysis, respectively.

First, we evaluated the differences in the composition of microbiota between the antrum and corpus using paired samples. In the $H$. pylori-negative group, there were no differences in the $\alpha$-diversity indices, including the number of observed OTUs and Chao1, ACE, and Shannon, between these two regions (Fig. 1). Similarly, $\beta$-diversity analysis using unweighted and weighted UniFrac data showed that the bacterial communities did not differ between the regions (Fig. 2). However, in H. pyloripositive patients, the number of observed OTUs and the Shannon index in the corpus was significantly lower than that in the antrum, with a non-significant decrease in the ing $\beta$-diversity of gastric bacterial communities between different $H$. pylori status groups using unweighted and weighted UniFrac distances

indices of Chaol and ACE in the corpus (Fig. 1). The relative abundance of $H$. pylori inversely correlated with the $\alpha$-diversity indices (Fig. 3). Additionally, the weighted UniFrac analysis revealed a significant difference in the gastric microbiota structure between the antrum and corpus in the pre-eradication patients (Fig. 2).

Next, we evaluated the changes in the gastric microbiota caused by $H$. pylori infection. Compared to the $H$. pylorinegative group, the $\alpha$-diversity of gastric microbiota was reduced in the pre-eradication group, as indicated by a significant reduction in the numbers of observed OTUs and Chao1, ACE, and Shannon indices in the antrum and corpus (Fig. 1). Unweighted and weighted UniFrac analyses showed that the gastric microbial community in the $H$. pylori-positive patients clustered separately from that in the $H$. pylori-negative patients (Fig. 2). 
Fig. 3 Correlation between the abundance of $H$. pylori and $\alpha$-diversity of the gastric microbiome. Correlation between the abundance of $H$. pylori and the number of observed OTUs (a), Chao1 index (b), ACE index (c), and Shannon index (d). Correlation was determined using Spearman's rank method
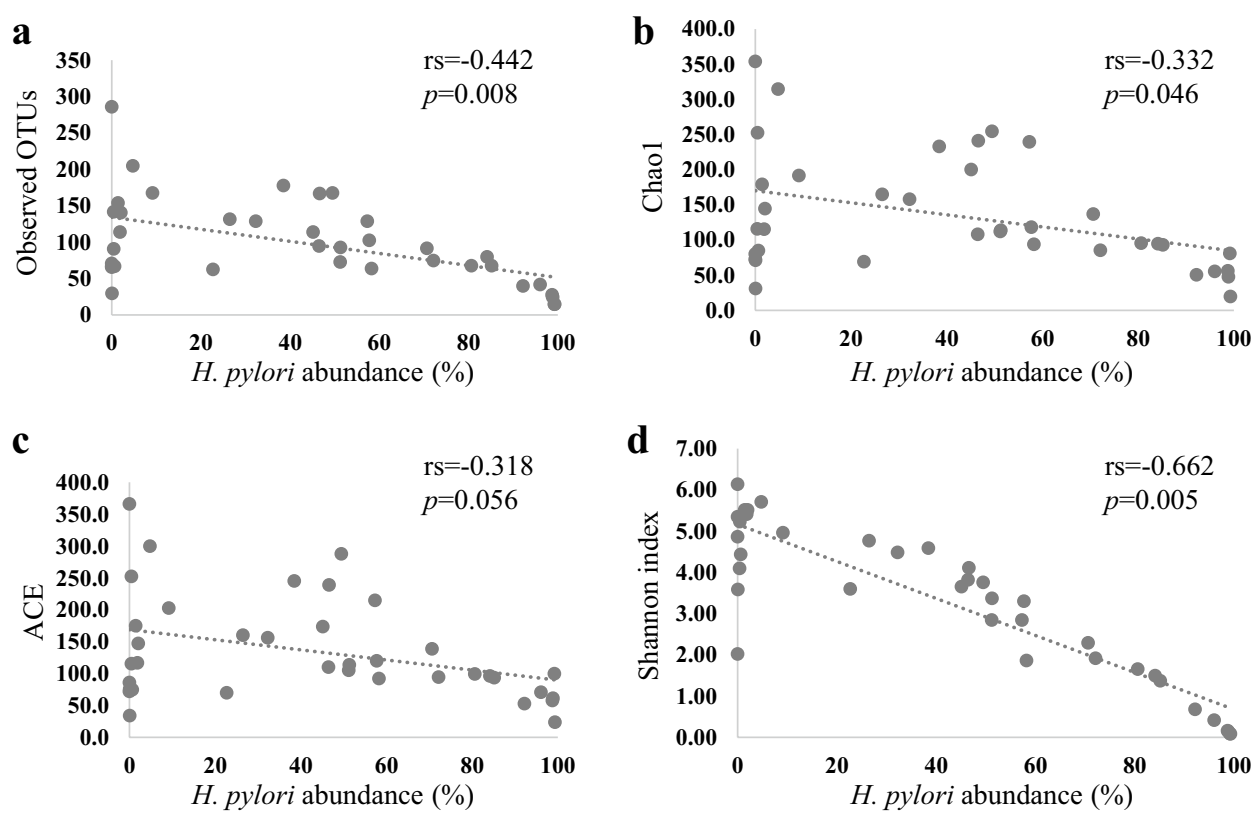

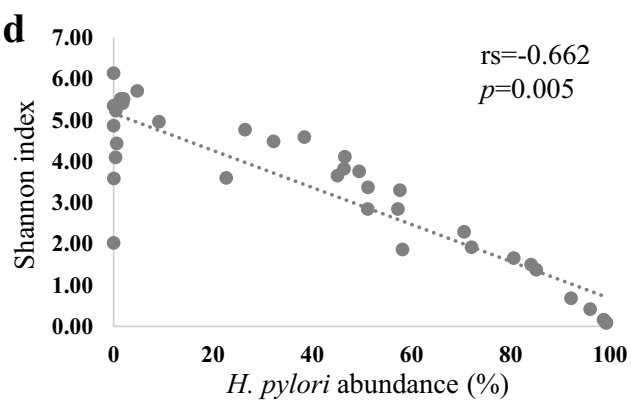

\section{Effect of $\boldsymbol{H}$. pylori eradication on the gastric microbiota}

Paired gastric samples from 19 patients with early GC were obtained after a median period of 13 months [range 8-24 months; $<12$ months $(n=3), 12-24$ months $(n=10),>24$ months $(n=6)]$ after successful eradication therapy. We detected H. pylori reads in six out of 19 antrum biopsy samples and eight out of 18 corpus biopsy samples obtained after eradication using 16S sequencing (Supplemental Table 1). However, the relative abundances of $H$. pylori in these samples were low (0.033-0.33\%). Previous studies have suggested a $1 \%$ cut-off for the positive readout for $H$. pylori colonization using pyrosequencing [21]. Taken together with the negative readout for histology and UBT for $H$. pyloripositive subjects after eradication therapy, we concluded that H. pylori was successfully eradicated in these patients.

H. pylori eradication significantly increased the Shannon index, but did not affect other $\alpha$-diversity metrics (Fig. 1, Supplemental Figure 1). Moreover, all metrics associated with $\alpha$-diversity in the antrum and corpus of patients in the post-eradication group were lower than those in the $H$. pylori-negative patients (Fig. 1). UniFrac analysis showed that the composition of the gastric bacterial community significantly changed after $H$. pylori eradication, but that in the post-eradication group did not return to normal as in the negative group. The gastric bacterial communities were clustered into three groups based on H. pylori status (Fig. 2).

\section{a- and $\beta$-diversity analysis by removing $H$. pylori reads}

Since the gastric microbial community in $H$. pylori-positive subjects was dominated by H. pylori (Supplemental Table 2), the difference in the composition of gastric microbiota between the negative and positive groups was not surprising. Therefore, we removed H. pylori reads from our sample data and reanalyzed the data. Most differences in the $\alpha$ - and $\beta$-diversity metrics of the gastric microbiota between $H$. pylori-negative and pre-eradication groups remained robust even after removing $H$. pylori reads (Supplemental Figure 2). In contrast, the difference in the $\alpha$-diversity metrics and dissimilarities in UniFrac analyses of the bacterial communities between pre- and post-eradication groups disappeared after omitting the $H$. pylori reads, except for the Shannon index and difference in weighted UniFrac in the corpus (Supplemental Figures 3, 4).

\section{Comparison of the microbiota composition between different $\boldsymbol{H}$. pylori status groups}

All the three groups of patients predominantly possessed the following phyla as part of their gastric microbiota: Firmicutes, Proteobacteria, Bacteroidetes, Actinobacteria, and Fusobacteria. Regardless of the presence of $H$. pylori, the antrum and corpus showed an abundance of Firmicutes, except for the corpus of the pre-eradication group wherein 


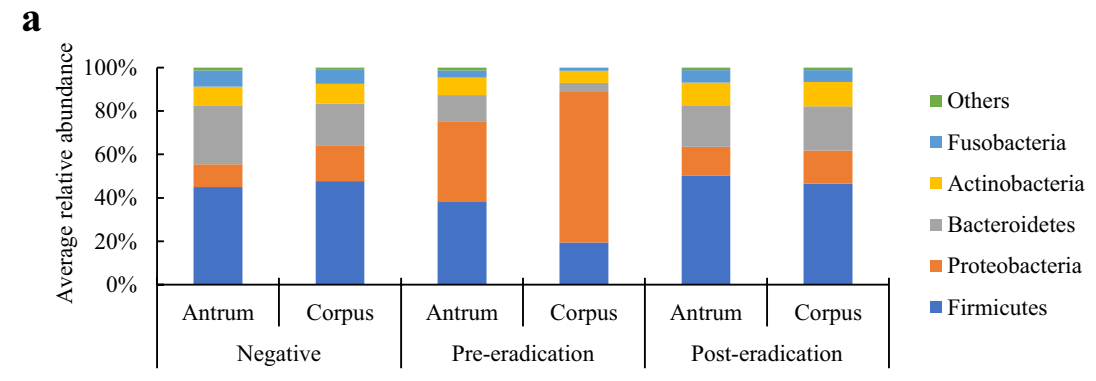

b
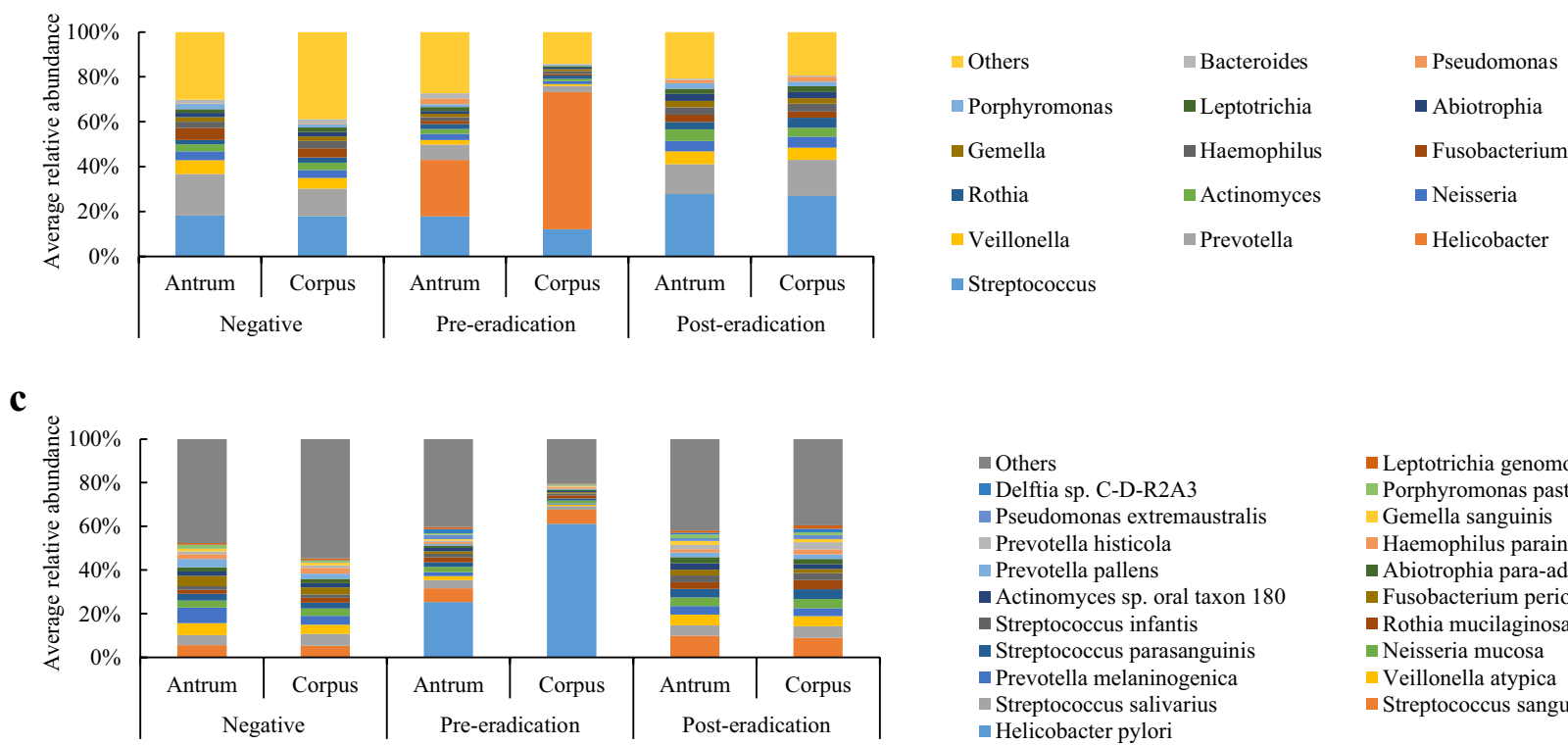
Others
Delftia sp. C-D-R2A3
- Pseudomonas extremaustralis
- Prevotella histicola
Prevotella pallens
- Actinomyces sp. oral taxon 180
- Streptococcus infantis
- Streptococcus parasanguinis
- Prevotella melaninogenica
- Streptococcus salivarius
- Helicobacter pylori

Fig. 4 Average relative abundances of taxa in the stomach of $H$. pylori-negative, pre-eradication, and post-eradication patients. Changes in the phylum (a), genus (b), and species (c)

Proteobacteria (including H. pylori) was predominant (Fig. 4, Supplemental Table 3). Among the phyla, Proteobacteria was significantly enriched in the corpus of $H$. pylori-positive patients as compared to that in $\mathrm{H}$. pylorinegative subjects, whereas the abundance of Bacteroidetes in the corpus and Firmicutes, Bacteroidetes, and Spirochetes in the antrum were significantly reduced (Supplemental Table 4). The eradication of $H$. pylori reduced the abundance of Proteobacteria and increased the abundance of Firmicutes, Bacteroidetes, and Fusobacteria in the gastric corpus. We found no significant differences in the relative abundance of bacteria in each phylum between the $H$. pylori-negative and post-eradication groups.

The heatmap showed the genera that were abundant in the $H$. pylori-negative, pre-eradication, and post-eradication samples (Fig. 5). As compared to the bacteria in the negative and pre-eradication groups, Helicobacter was the only genus that was enriched in the pre-eradication group, whereas numerous genera, including Veillonella, Bacteroides, Prevotella, Lactobacillus, Blautia, Ralstonia, Faecalibacterium, Methylobacterium, and Megamonas, were enriched in the $H$. pylori-negative patients (Supplemental Tables 5, 6). We also observed a significant enrichment in the abundance of five genera (Blautia, Ralstonia, Faecalibacterium, Methylobacterium, and Megamonas) in the $H$. pylori-negative patients as compared to the genera in the post-eradication group. $H$. pylori eradication markedly reduced the abundance of the genus Helicobacter and increased the abundance of several genera, including Prevotella, Veillonella, Actinomyces, and Solobacterium (Fig. 5, Supplemental Table 6). Furthermore, there were significant differences in the relative abundances of 8 and 23 species in the antrum and corpus, respectively, between $H$. pylori-negative and pre-eradication groups. All the taxa, excluding $H$. pylori, were enriched in the negative group (Fig. 5, Supplemental Tables 7, 8). As compared to the microbial population in the post-eradication group, we identified ten and fifteen species in the antrum and corpus, respectively, that were enriched in the negative group. Among these species, Ralstonia sp. W-15 was the most enriched in the corpus, with an average abundance of $4.66 \%$. Successful eradication of $H$. pylori significantly altered the abundance of 12 species. As expected, this reduced the 


\section{Genus level}

Species level

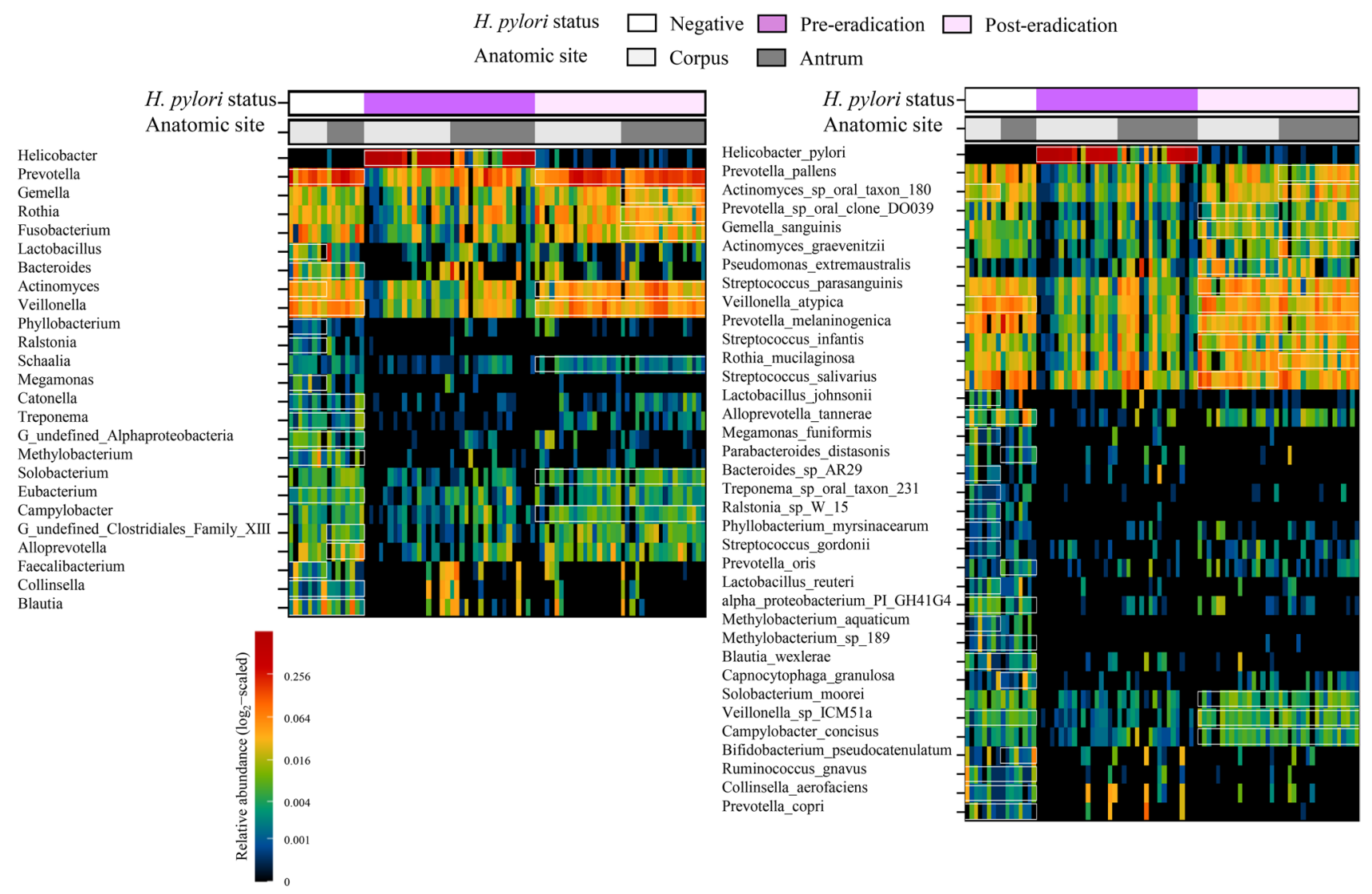

Fig. 5 Heatmap of the relative abundance of gastric taxa stratified by H. pylori status and gastric site. Wilcoxon rank sum test was used to compare the relative abundances of gastric taxa in $H$. pylori-negative

abundance of $H$. pylori from $25.295 \%$ to 0.033 and 61.148 to $0.044 \%$ in the antrum and corpus, respectively (Supplemental Table 7). The other species belonging to the genera Streptococcus, Veillonella, Prevotella, Gemella, Pseudomonas, Solobacterium, and Campylobacter increased after the eradication of $H$. pylori (Fig. 5, Supplemental Table 8).

\section{Discussion}

Previous studies have demonstrated that $H$. pylori infection is associated with marked changes in the gastric microbiota [14, 19, 22-24]. Here, we confirmed that $H$. pylori-negative and -positive patients had different bacterial profiles characterized by less richness of gastric microbiota and the reduction in abundances of Blautia, Ralstonia, Faecalibacterium, Methylobacterium, and Megamonas in $H$. pylori-positive patients. Furthermore, there were significant differences in the $\alpha$ - and $\beta$-diversities between these two patient groups even after removing the $H$. pylori-related OTUs, indicating that changes in the gastric microbiota in patients with those in pre-eradication or post-eradication patients. The Wilcoxon signed-rank test was used to compare the difference in paired samples from pre- and post- $H$. pylori eradication groups

the $H$. pylori-positive patients were not merely due to the presence of $H$. pylori. Dysbiosis persisted for a long time after eradicating $H$. pylori.

Studies have reported the restoration of the richness and evenness of gastric microbiota to a status similar to that of the negative subjects by the eradication $[22,23]$; however, we failed to demonstrate marked effects of $H$. pylori eradication on microbial diversity. Among the $\alpha$-diversity metrices, the Shannon index accounts for richness and evenness of species, while the remaining three metrices represented richness only. If some species are much more abundant than others, the Shannon index is low, thereby accounting for the increase in the Shannon index observed upon the eradication of $H$. pylori. In contrast, our findings showed that the eradication of $H$. pylori did not affect the number of OTUs and Chao 1 and ACE indices. Moreover, these metrices were lower in the eradicated subjects than those in the H. pylorinegative subjects, suggesting that $H$. pylori eradication may be less effective in improving the richness of gastric microbiota in patients with early GC. Li et al. reported that H. pylori eradication normalized the composition of the 
microbial community of the stomach [23]. Guo et al. [22] demonstrated a significant change in $\beta$-diversity after the eradication of $H$. pylori, as assessed using UniFrac analysis. However, the composition of the microbial community in the eradicated subjects was significantly different from that in $H$. pylori-negative patients. Furthermore, we found that the microbiome profiles differed between the antrum and corpus in the H. pylori-positive group, with less bacterial richness in the corpus, although this difference was not observed in other groups. These findings were also contrary to previous studies that reported that paired tissue samples from the antrum and corpus had similar bacterial composition and diversity, regardless of the presence of $H$. pylori $[23,25,26]$. This can be attributed to the differences in the methodologies used in published literature and this study. However, we believe that the strict patient enrollment protocol employed in this study has resulted in significant differences in the microbial population and richness according to $H$. pylori colonization status and gastric anatomic sites. In this study, only subjects who met all the five selection criteria were enrolled in the $H$. pylori-negative group; thus, this group comprised only $H$. pylori-naïve subjects. The majority of the published studies enrolled patients who were negative for the $H$. pylori antibody, rapid urease test, or UBT as part of the H. pylori-negative cohort. Therefore, this group may include patients after eradication or after spontaneous clearance of $H$. pylori as well as $H$. pylori-naïve individuals, suggesting the heterogeneity of the population. Furthermore, we only enrolled patients who underwent ESD for early GC in the H. pylori-positive group. Gastric microbiota changes during the progression of $H$. pylori-induced gastritis to atrophic gastritis, intestinal metaplasia, and GC $[23,27]$. Wang et al. reported that the changing patterns of the composition and function of the gastric microbiota are highly indicative of the stages of neoplastic progression in the stomach [27]. Thus, the gastric microbiota and how $H$. pylori eradication affects these profiles in patients with different $H$. pylori-induced pathologies should be analyzed independently. Moreover, $H$. pylori prefers to colonize the normal gastric mucosa and gradually decreases in abundance during the progression of gastritis from the antrum to the corpus before finally disappearing. The average abundance of $H$. pylori in the antrum and corpus was $22.3 \%$ and $61.1 \%$, respectively, in our patients, who exhibited moderate to severe atrophic gastritis. Since the $\alpha$-diversity matrices were inversely correlated with the abundance of $H$. pylori, the differences in the diversity of the microbiota in our patients may be due to the predominance of $H$. pylori in the corpus. To the best of our knowledge, this is the first report on the incomplete reversal of gastric dysbiosis on the eradication of $H$. pylori in early GC patients who have a high risk for metachronous GC even after successful eradication [28]
Experimental studies have suggested that microbes other than $H$. pylori may also be involved in gastric carcinogenesis [11-13], although direct clinical evidence for a link between specific dysbiosis or bacterial taxa and the development of GC remains to be uncovered. Hsieh et al. reported an increased abundance of Clostridium and Fusobacterium in GC tissues [29]. Ferreira et al. demonstrated that the microbiota in GC tissues was characterized by the reduction in the abundance of Helicobacter and over-representation of intestinal commensals, including Citrobacter, Clostridium, Lactobacillus, Achromobacter, and Rhodococcus [30]. In contrast, Coker et al. observed the significant enrichment of the oral microbiome, including Parvimonas micra, Peptostreptococcus stomatis, Fusobacterium nucleatum, and Gemella in GC tissues compared to their abundance in the precancerous tissues [31]. Thus, the data were discordant, but microbial populations show alterations, although not identical in all patients, in GC tissues. More importantly, several studies have shown that the paired tumor and nontumor tissue samples had similar microbiota profiles [23, 27, 32], suggesting the importance of the dysbiosis of background mucosa in the GC development. Compared to the negative group of patients, only Helicobacter was significantly enriched, while more than ten taxa were significantly reduced in the pre-eradication group. Among these reduced taxa, Blautia, Ralstonia, Faecalibacterium, Methylobacterium, and Megamonas were also found to be more deleted in the post-eradication group as compared to the negative group. Therefore, the deletion of these taxa may be involved as a common mechanism for primary and metachronous GC after the eradication of $H$. pylori. Anti-inflammatory effects have been demonstrated for Blautia and Faecalibacterium in gastrointestinal diseases, including inflammatory bowel diseases and intestinal graft-versus-host disease [33-36]. Interestingly, colorectal cancer tissues show a reduction in the abundance of these taxa [37,38]. Taken together, our data and that previously published suggest that reduced abundances of Blautia and Faecalibacterium may maintain or enhance inflammation in H. pylori-infected and -eradicated gastric mucosa, thereby leading to the development of GC. Ralstonia, a Gram-positive bacterium, is enriched in the gastric mucosa of patients with GC before subtotal gastrectomy [39] and those with gastric inflammation one year after eradication therapy [40]. Thus, the reduction of Ralstonia is unlikely to be linked to GC. The pathogenicity of Methylobacterium and Megamonas in the stomach has not been studied; however, they may not be crucial during gastric carcinogenesis since they are found at an average abundance of $<1 \%$.

This study has some limitations. First, we used a small patient cohort. Larger sample sizes will help detect sensitive differences in the microbiota between groups. Our strict 
criteria for the enrollment of $H$. pylori-naïve and $H$. pyloripositive patients with early GC and analysis of bacteria using samples obtained from the same site in the stomach will reduce intra-group variation and minimize the bias associated with small cohorts. Second, we were unable to match the patients in $H$. pylori-negative and -positive patients based on age and sex. However, several studies have detected no association between gastric microbial features and patient age or sex $[23,26]$. Thus, we believe this would not majorly affect this study. Third, we enrolled patients who underwent ESD for early GC since they were at high risk for developing GC even after successful eradication of $H$. pylori. However, during the follow-up period, no patient metachronously developed GC, suggesting the validation of the study findings in patients with metachronous GC. Finally, the followup period after eradication therapy was not the same for all the patients; a majority of the patients were followed up for $>12$ months, with the shortest period being 8 months. The effects of the eradication of $H$. pylori on gastric microbiota were assessed after a sufficient interval.

In conclusion, gastric dysbiosis may persist for a long time after the successful eradication of $H$. pylori in patients with a history of GC. Alterations in the gastric microbiota may form the underlying mechanism involved in the development of metachronous GC after the eradication of $H$. pylori as well as primary GC.

Acknowledgements This work was partially supported by the JSPS KAKENHI (Grant Number JP25460932).

Author contributions All authors commented on previous versions of the manuscript. All authors read and approved the final manuscript. Conceptualization: TW, YN, TT, and YF. Methodology: TW, YN, WS and $\mathrm{MH}$. Formal analysis: TW, WS, GN, and MH. Investigation: TW, AH, KO, SF, SH, FT, YN, KT, and TT. Writing-original draft: TW visualization: TW, AH, GN, and WS supervision: $\mathrm{MH}$ and YF. Funding acquisition: TW, and YF.

\section{Compliance with ethical standards}

Conflict of interest The authors declare that they have no conflict of interest.

Human rights statement All procedures followed were in accordance with the ethical standards of the responsible committee on human experimentation (institutional and national) and with the Helsinki Declaration of 1964 and later versions. Informed consent to be included in the study, or the equivalent, was obtained from all patients.

Open Access This article is licensed under a Creative Commons Attribution 4.0 International License, which permits use, sharing, adaptation, distribution and reproduction in any medium or format, as long as you give appropriate credit to the original author(s) and the source, provide a link to the Creative Commons licence, and indicate if changes were made. The images or other third party material in this article are included in the article's Creative Commons licence, unless indicated otherwise in a credit line to the material. If material is not included in the article's Creative Commons licence and your intended use is not permitted by statutory regulation or exceeds the permitted use, you will need to obtain permission directly from the copyright holder. To view a copy of this licence, visit http://creativecommons.org/licenses/by/4.0/.

\section{References}

1. Nomura A, Stemmermann GN, Chyou PH, Kato I, Perez-Perez GI, Blaser MJ. Helicobacter pylori infection and gastric carcinoma among Japanese Americans in Hawaii. N Engl J Med. 1991;325(16):1132-6.

2. Parsonnet J, Friedman GD, Vandersteen DP, Chang Y, Vogelman $\mathrm{JH}$, Orentreich $\mathrm{N}$, et al. Helicobacter pylori infection and the risk of gastric carcinoma. N Engl J Med. 1991;325(16):1127-31.

3. Plummer M, Franceschi S, Vignat J, Forman D, de Martel C. Global burden of gastric cancer attributable to Helicobacter pylori. Int J Cancer. 2015;136(2):487-90.

4. Ma JL, Zhang L, Brown LM, Li JY, Shen L, Pan KF, et al. Fifteenyear effects of Helicobacter pylori, garlic, and vitamin treatments on gastric cancer incidence and mortality. J Natl Cancer Inst. 2012;104(6):488-92.

5. Fukase K, Kato M, Kikuchi S, Inoue K, Uemura N, Okamoto S, et al. Effect of eradication of Helicobacter pylori on incidence of metachronous gastric carcinoma after endoscopic resection of early gastric cancer: an open-label, randomised controlled trial. Lancet. 2008;372(9636):392-7.

6. Choi IJ, Kook MC, Kim YI, Cho SJ, Lee JY, Kim CG, et al. Helicobacter pylori therapy for the prevention of metachronous gastric cancer. N Engl J Med. 2018;378(12):1085-95.

7. Lee YC, Chiang TH, Chou CK, Tu YK, Liao WC, Wu MS, et al. Association between Helicobacter pylori eradication and gastric cancer incidence: a systematic review and meta-analysis. Gastroenterology. 2016;150(5):1113-24 e5.

8. Bae SE, Choi KD, Choe J, Kim SO, Na HK, Choi JY, et al. The effect of eradication of Helicobacter pylori on gastric cancer prevention in healthy asymptomatic populations. Helicobacter. 2018;23(2):e12464.

9. Take S, Mizuno M, Ishiki K, Yoshida T, Ohara N, Yokota K, et al. The long-term risk of gastric cancer after the successful eradication of Helicobacter pylori. J Gastroenterol. 2011;46(3):318-24.

10. Maeda M, Yamashita S, Shimazu T, Iida N, Takeshima H, Nakajima T, et al. Novel epigenetic markers for gastric cancer risk stratification in individuals after Helicobacter pylori eradication. Gastric Cancer. 2018;21(5):745-55.

11. Wang TC, Dangler CA, Chen D, Goldenring JR, Koh T, Raychowdhury R, et al. Synergistic interaction between hypergastrinemia and Helicobacter infection in a mouse model of gastric cancer. Gastroenterology. 2000;118(1):36-47.

12. Lofgren JL, Whary MT, Ge Z, Muthupalani S, Taylor NS, Mobley $\mathrm{M}$, et al. Lack of commensal flora in Helicobacter pylori-infected INS-GAS mice reduces gastritis and delays intraepithelial neoplasia. Gastroenterology. 2011;140(1):210-20.

13. Lertpiriyapong K, Whary MT, Muthupalani S, Lofgren JL, Gamazon ER, Feng Y, et al. Gastric colonisation with a restricted commensal microbiota replicates the promotion of neoplastic lesions by diverse intestinal microbiota in the Helicobacter pylori INS-GAS mouse model of gastric carcinogenesis. Gut. 2014;63(1):54-63.

14. Parsons BN, Ijaz UZ, D’Amore R, Burkitt MD, Eccles R, Lenzi L, et al. Comparison of the human gastric microbiota in hypochlorhydric states arising as a result of Helicobacter pylori-induced 
atrophic gastritis, autoimmune atrophic gastritis and proton pump inhibitor use. PLoS Pathog. 2017;13(11):e1006653.

15. Zhao Y, Gao X, Guo J, Yu D, Xiao Y, Wang H, et al. Helicobacter pylori infection alters gastric and tongue coating microbial communities. Helicobacter. 2019;24(2):e12567.

16. Mori G, Nakajima T, Asada K, Shimazu T, Yamamichi N, Maekita $\mathrm{T}$, et al. Incidence of and risk factors for metachronous gastric cancer after endoscopic resection and successful Helicobacter pylori eradication: results of a large-scale, multicenter cohort study in Japan. Gastric Cancer. 2016;19(3):911-8.

17. Kimura K, Takemoto T. An endoscopic recognition of the atrophic border and its significance in chronic gastritis. Endoscopy. 1969;1(03):87-97.

18. Nadatani Y, Watanabe T, Suda W, Nakata A, Matsumoto Y, Kosaka S, et al. Gastric acid inhibitor aggravates indomethacininduced small intestinal injury via reducing Lactobacillus johnsonii. Sci Rep. 2019;9(1):17490.

19. Andersson AF, Lindberg M, Jakobsson H, Backhed F, Nyren P, Engstrand L. Comparative analysis of human gut microbiota by barcoded pyrosequencing. PLoS ONE. 2008;3(7):e2836.

20. Iwasawa K, Suda W, Tsunoda T, Oikawa-Kawamoto M, Umetsu $\mathrm{S}$, Takayasu L, et al. Dysbiosis of the salivary microbiota in pediatric-onset primary sclerosing cholangitis and its potential as a biomarker. Sci Rep. 2018;8(1):5480.

21. Kim J, Kim N, Jo HJ, Park JH, Nam RH, Seok YJ, et al. An appropriate cutoff value for determining the colonization of Helicobacter pylori by the pyrosequencing method: comparison with conventional methods. Helicobacter. 2015;20(5):370-80.

22. Guo Y, Zhang Y, Gerhard M, Gao JJ, Mejias-Luque R, Zhang L, et al. Effect of Helicobacter pylori on gastrointestinal microbiota: a population-based study in Linqu, a high-risk area of gastric cancer. Gut. 2020;69(9):1598-607.

23. Li TH, Qin Y, Sham PC, Lau KS, Chu KM, Leung WK. Alterations in gastric microbiota after $H$. pylori eradication and in different histological stages of gastric carcinogenesis. Sci Rep. 2017;7:44935.

24. Miftahussurur M, Waskito LA, El-Serag HB, Ajami NJ, Nusi IA, Syam AF, et al. Gastric microbiota and Helicobacter pylori in Indonesian population. Helicobacter. 2020;25(4):e12695.

25. Bik EM, Eckburg PB, Gill SR, Nelson KE, Purdom EA, Francois F, et al. Molecular analysis of the bacterial microbiota in the human stomach. Proc Natl Acad Sci USA. 2006;103(3):732-7.

26. Yu G, Torres J, Hu N, Medrano-Guzman R, Herrera-Goepfert R, Humphrys MS, et al. Molecular characterization of the human stomach microbiota in gastric cancer patients. Front Cell Infect Microbiol. 2017;7:302.

27. Wang Z, Gao X, Zeng R, Wu Q, Sun H, Wu W, et al. Changes of the gastric mucosal microbiome associated with histological stages of gastric carcinogenesis. Front Microbiol. 2020;11:997.

28. Xiao S, Li S, Zhou L, Jiang W, Liu J. Helicobacter pylori status and risks of metachronous recurrence after endoscopic resection of early gastric cancer: a systematic review and meta-analysis. J Gastroenterol. 2019;54(3):226-37.

29. Hsieh YY, Tung SY, Pan HY, Yen CW, Xu HW, Lin YJ, et al. Increased abundance of clostridium and fusobacterium in gastric microbiota of patients with gastric cancer in Taiwan. Sci Rep. 2018;8(1):158.

30. Ferreira RM, Pereira-Marques J, Pinto-Ribeiro I, Costa JL, Carneiro F, Machado JC, et al. Gastric microbial community profiling reveals a dysbiotic cancer-associated microbiota. Gut. 2018;67(2):226-36

31. Coker OO, Dai Z, Nie Y, Zhao G, Cao L, Nakatsu G, et al. Mucosal microbiome dysbiosis in gastric carcinogenesis. Gut. 2018;67(6):1024-32.

32. Wu ZF, Zou K, Wu GN, Jin ZJ, Xiang CJ, Xu S, et al. A comparison of tumor-associated and non-tumor-associated gastric microbiota in gastric cancer patients. Dig Dis Sci. 2020. https:// doi.org/10.1007/s10620-020-06415-y.

33. Zhou L, Zhang M, Wang Y, Dorfman RG, Liu H, Yu T, et al. Faecalibacterium prausnitzii produces butyrate to maintain Th17/ Treg balance and to ameliorate colorectal colitis by inhibiting histone deacetylase 1. Inflamm Bowel Dis. 2018;24(9):1926-40.

34. Lopez-Siles M, Martinez-Medina M, Abella C, Busquets D, Sabat-Mir M, Duncan SH, et al. Mucosa-associated Faecalibacterium prausnitzii phylotype richness is reduced in patients with inflammatory bowel disease. Appl Environ Microbiol. 2015;81(21):7582-92.

35. Jenq RR, Taur Y, Devlin SM, Ponce DM, Goldberg JD, Ahr $\mathrm{KF}$, et al. Intestinal blautia is associated with reduced death from graft-versus-host disease. Biol Blood Marrow Transplant. 2015;21(8):1373-83.

36. Benitez-Paez A, Gomez Del Pugar EM, Lopez-Almela I, MoyaPerez A, Codoner-Franch P, Sanz Y. Depletion of Blautia species in the microbiota of obese children relates to intestinal inflammation and metabolic phenotype worsening. mSystems. 2020;5(2):e00857.

37. Chen W, Liu F, Ling Z, Tong X, Xiang C. Human intestinal lumen and mucosa-associated microbiota in patients with colorectal cancer. PLoS ONE. 2012;7(6):e39743.

38. Shah MS, DeSantis T, Yamal JM, Weir T, Ryan EP, Cope JL, et al. Re-purposing 16S rRNA gene sequence data from within case paired tumor biopsy and tumor-adjacent biopsy or fecal samples to identify microbial markers for colorectal cancer. PLoS ONE. 2018;13(11):e0207002.

39. Tseng CH, Lin JT, Ho HJ, Lai ZL, Wang CB, Tang SL, et al. Gastric microbiota and predicted gene functions are altered after subtotal gastrectomy in patients with gastric cancer. Sci Rep. 2016;6:20701.

40. Sung JJY, Coker OO, Chu E, Szeto CH, Luk STY, Lau HCH, et al. Gastric microbes associated with gastric inflammation, atrophy and intestinal metaplasia 1 year after Helicobacter pylori eradication. Gut. 2020;69(9):1572-80.

Publisher's Note Springer Nature remains neutral with regard to jurisdictional claims in published maps and institutional affiliations. 